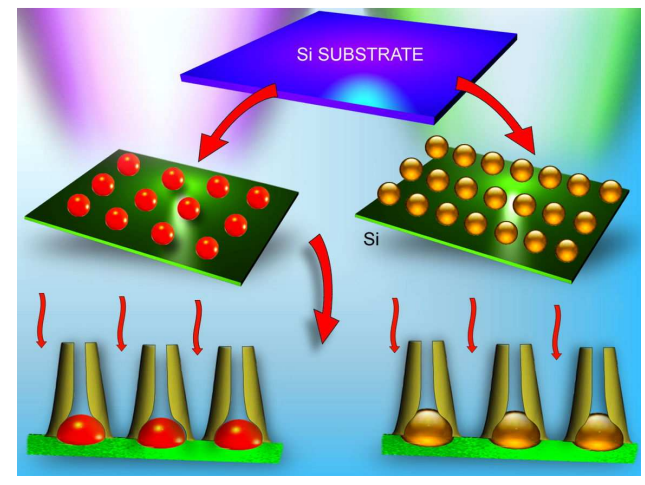




\title{
Carbon nanoflake-nanoparticle interface: a comparative study on structure and photoluminescent properties of carbon nanoflakes synthesized on nanostructured gold and carbon by hot filament CVD
}

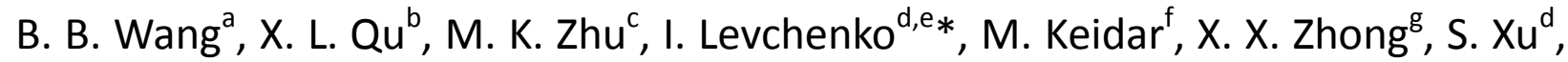 \\ K. Ostrikov ${ }^{\mathrm{e}, \mathrm{h}, \mathrm{i}}$
}

${ }^{a}$ College of Chemistry and Chemical Engineering, Chongqing University of Technology, 69 Hongguang Rd, Lijiatuo, Banan District, Chongqing 400054, P. R. China.

${ }^{b}$ Institute of Microstructure and Properties of Advanced Materials, Beijing University of Technology, Beijing 100124, P. R. China.

${ }^{c}$ College of Materials Science and Engineering, Beijing University of Technology, Beijing 100124, P. R. China.

${ }^{d}$ Plasma Sources and Applications Centre, National Institute of Education, Nanyang Technological University, 1 Nanyang Walks, 637616, Singapore

${ }^{e}$ School of Chemistry, Physics and Mechanical Engineering, Queensland University of Technology, Brisbane QLD 4000, Australia.

${ }^{f}$ Mechanical and Aerospace Engineering, The George Washington University, Washington DC 20052, USA.

${ }^{g}$ Department of Physics and Astronomy, Shanghai Jiao Tong University, Shanghai 200240, P. R. China

${ }^{h}$ CSIRO-QUT Joint Sustainable Materials and Devices Laboratory, Commonwealth Scientific and Industrial Research Organization, P.O. Box 218, Lindfield NSW 2070, Australia.

${ }^{i}$ Plasma Nanoscience, School of Physics, The University of Sydney, Sydney NSW 2006, Australia.

\footnotetext{
* Corresponding author at: School of Chemistry, Physics and Mechanical Engineering, Queensland University of Technology, Brisbane QLD 4000, Australia. Tel.: +61 029413 7634. E-mail address: Igor.Levchenko@qut.edu.au (I. Levchenko).
} 


\begin{abstract}
Three dimensional vertically-oriented carbon nanoflakes grown on carbon and gold nanoparticles by the hot filament chemical vapor deposition in $\mathrm{CH}_{4}$ environment demonstrate quite similar structure and composition, but drastically different room temperature photoluminescent properties. The interfacial interactions were asserted to be the main reason for the differences in the optical emission. The mechanisms of highly oriented growth, generation and enhancement of photoluminescence were investigated, and it was demonstrated that the formation of oriented nanoflakes resulted from the stress produced in the carbon layers on carbon and gold nanoparticles. Specifically, deformation of nanoparticles and difference in the expansion rates of carbon layer, gold and carbon nanoparticles are the main causes for the stress formation. The oriented growth of carbon nanoflakes is maintained by the repulsion effect between the carbon nanoflakes due to the net charge produced from the hydrocarbon radicals on the edges of carbon nanoflakes via charge transfer between $\mathrm{H}$ and $\mathrm{C}$ atoms. The photoluminescence generation of carbon nanoflakes is related to the $s p^{2}$ carbon clusters on the edges of carbon nanoflakes. Strong green photoluminescent emission from the carbon nanoflake/gold nanoparticle system than from the carbon nanoflake/carbon nanoparticle system is the result of the intense plasmon emission from gold nanoparticles.
\end{abstract}




\section{Introduction}

Graphene and graphene-related nanomaterials feature unique structure and many important properties that warrant their application in various areas of optoelectronics, nanoelectronics, sensors, green energy power source, medicine and other fields [1-5]. Due to these reasons, the graphene-related nanomaterials such as graphene nanoflakes, graphene networks and graphene nanodots are attracting an increasing interest in last years [6-9].

Recently, hybrid nanomaterials and complex nanostructures composed of graphene and carbon nanotubes interfaced with composites and metal nanoparticles (NPs) (inclusion noble metals such as $\mathrm{Ag}$ and $\mathrm{Au}$ ) have become the important research areas due to their potential applications in optoelectronics, biology, and medical sciences such as cell imaging, drug delivery and cell therapy [5,10-15]. However, the hybrid nanomaterials of graphene and noble metal NPs are mainly synthesized via solution routes, which require a long (up to $24 \mathrm{~h}$ ) synthesis duration due to the complicated reactions involved $[13,15]$. Importantly, advanced applications of the hybrid nanomaterials of graphene and noble metal NPs are often related to their optical properties [5,11-15], but the effects of the interfaces between graphene and noble metal NPs on the optical emission is not comprehensively studied. For example, the results obtained by Lan $e t$ al. and the studies of Park et al. indicate that the photoluminescence (PL) emission of graphene oxide/Ag and graphene quantum dots/Au hybrid nanomaterials is enhanced by $\mathrm{Ag}$ and $\mathrm{Au}$ nanoparticles, and the enhancement was attributed to surface plasmons generated by $\mathrm{Ag}$ and $\mathrm{Au}$ NPs $[12,15]$. Furthermore, there are reports that the charge and energy transfer occurs in the interfaces between the graphene and gold nanoparticles, thus enhancing the surface Raman scattering [16,17]. However, the effects of charge and energy transfer on the photoluminescence (PL) of graphene/metal hybrid nanomaterials have seldom been reported. It is known that the charge and energy transfer produced in the interfaces has important influences on the photoluminescent emission of hybrid nanomaters, thus the PL mechanism of hybrid nanomaterials composed of graphene and noble metal requires further research for their applications. This work mainly aims to study the PL properties of carbon nanoflakes and noble metal hybrid nanomaterials through their interface structures.

In some previous works $[18,19]$, graphene films and nanoflakes were reported to directly grow on $\mathrm{Cu}$ foils or Au NPs by chemical vapor deposition (CVD). This apparently implies that 
the present techniques used for the preparation of hybrid graphene-metal nanomaterials by CVD can be simplified, and thus the interfacial structures between graphene and metal NPs should be further studied by characterization instruments such as high-resolution transmission electron microscope and other sophisticated characterization techniques.

In this work, we have synthesized hybrid nanomaterials composed of carbon nanoflakes (CNFs) and Au/carbon NPs by hot filament CVD (HFCVD) according to the methods described in references [6] and [19]. In the HFCVD technique, high temperature of filaments $\left(\sim 1800{ }^{\circ} \mathrm{C}\right)$ ensures fast decomposition of reactive gases in the process chamber, and the substrates can be efficiently heated by the thermal radiation of hot filaments installed at a short distance to the substrate. Moreover, high temperature difference between the filaments and substrate can intensify the diffusion of chemical radicals toward the substrate and thus enhance the nanomaterial growth rate [20]; our experiments have also demonstrated that indeed, the HFCVD technique is quite efficient in synthesizing the hybrid CNFs containing nanomaterials $[6,19]$. In particular, $\mathrm{CH}_{4}$ was directly supplied to the $\mathrm{CVD}$ chamber without other carrier gas to ensure the fast absorption of $\mathrm{CH}_{4}$ on the $\mathrm{Au} /$ carbon NP, because it benefits the layer-by-layer growth [21]. Furthermore, it was found that the oriented CNFs were grown in HFCVD system which differs from the plasma-enhanced CVD (PECVD) system. In PECVD system, the formation of vertically oriented graphene is related to the stress in graphene sheets and electric field built up by plasma [22-24]; however, there is no electric filed in the HFCVD system, and the formation and growth of oriented CNFs are related to the interfaces between carbon sheets and nanoparticles and the polar chemical radicals deposited on the edges of CNFs.

In the previous work [6], the PL properties of oriented CNFs were studied, but the origination of PL bands was not well understood. Specifically, the effects of interfaces of CNFs with metal nanodots on the PL properties of CNFs are seldom studied. Beginning with the PL emission of amorphous carbon nanodots, the origination of PL bands of oriented CNFs was studied here systematically, and found to be related to the edges of CNFs. Furthermore, the effects of interfaces between CNFs and Au NPs on PL properties of CNF system are studied.

Our results are important to control the structure of hybrid graphene-metal nanomaterials and nanostructures, understand the optical properties of graphene-related nanomaterials, and contribute to the development of optoelectronic devices of graphene-based nanomaterials. 


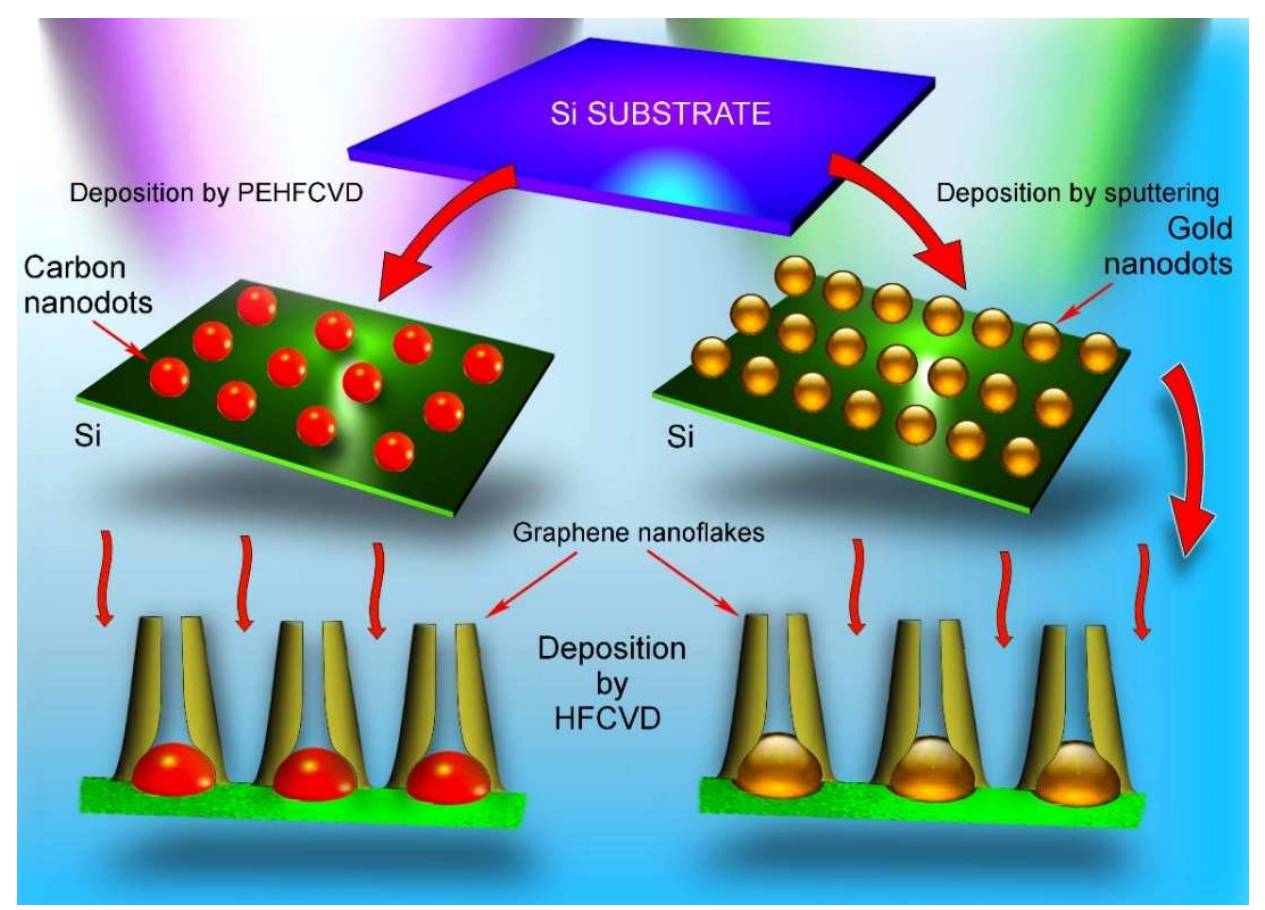

Fig. 1. Schematic diagram of synthesizing carbon nanoflakes. Deposition by PEHFCVD and by sputtering was used to form arrays of carbon and gold nanodots on Si substrates. Then, deposition by HFCVD was used to nucleate and grow vertically-aligned carbon nanoflakes on both carbon and gold nanodots.

\section{Experimental details and characterization}

The procedure of nanomaterial synthesis is sketched in Fig. 1. To form the CNF structure, $15 \mathrm{~nm}$ $\mathrm{Au}$ film and amorphous carbon NPs were pre-deposited on clean silicon substrates in magnetron sputtering system and plasma-enhanced hot filament CVD (PEHFCVD) system, respectively. Before synthesis, silicon substrates were chemically cleaned by ultrasonication in solution of toluene, acetone and ethanol, and then boiled in the solution composed of $\mathrm{NH}_{3} \cdot \mathrm{H}_{2} \mathrm{O}, \mathrm{H}_{2} \mathrm{O}_{2}$ and deionized water as described in Ref. (6).

The syntheses of amorphous carbon NPs and CNFs were carried out in PEHFCVD system described in detail elsewhere [6]. In the CVD chamber, three coiled tungsten filaments were installed at about $10 \mathrm{~mm}$ from the substrate to decompose the reaction gases and heat the substrate surface. The plasma was produced in the chamber by a DC bias power supply, with positive and negative electrodes connected to the filaments and substrate through a Mo holder, respectively.

When the amorphous carbon NPs were deposited on the silicon substrate, $\mathrm{N}_{2}$ and $\mathrm{H}_{2}$ were supplied into the CVD chamber at the same flow rate of $50 \mathrm{sccm}$ after vacuuming the CVD chamber to the pressure lower than $2 \mathrm{~Pa}$. Once the pressure was stabilized to about $2 \times 10^{3} \mathrm{~Pa}$ 
through adjusting the vacuum valve, the filaments were heated by $\mathrm{AC}$ current to $\sim 1800{ }^{\circ} \mathrm{C}$. Due to a short distance between the silicon substrate and filaments, the silicon substrate was fast heated to $850{ }^{\circ} \mathrm{C}$ by the thermal radiation of filaments. At this moment, the DC bias power supply was turned on to ignite the plasma, and the silicon substrate was treated for 5 min in $\mathrm{N}_{2}-\mathrm{H}_{2}$ plasma.

Table 1. Substrate, gas flow rate, time $(t)$ of growing samples A-D.

\begin{tabular}{cccc}
\hline Sample & Substrate & $\mathbf{C H}_{\mathbf{4}}(\mathbf{s c c m})$ & $\boldsymbol{t}$ (min) \\
\hline A & Carbon dots/Si & 70 & 7 \\
B & $\mathrm{Au} / \mathrm{Si}$ & 70 & 7 \\
C & Carbon dots/Si & 70 & 10 \\
D & $\mathrm{Au} / \mathrm{Si}$ & 70 & 10 \\
\hline
\end{tabular}

Then the $\mathrm{CH}_{4}$ was fed into the CVD chamber, the flow rates of $\mathrm{CH}_{4}, \mathrm{~N}_{2}$ and $\mathrm{H}_{2}$ were adjusted to 20, 30 and $50 \mathrm{sccm}$, and the amorphous carbon NPs were grown for $15 \mathrm{~min}$ in $\mathrm{CH}_{4}-\mathrm{N}_{2}-\mathrm{H}_{2}$ plasma under a bias current of $160 \mathrm{~mA}$.

The synthesis of CNFs was similar to the growth of amorphous carbon NPs. Silicon substrates with amorphous carbon NPs or Au film were installed in the CVD chamber, the pressure was lowered to less than $2 \mathrm{~Pa}$, and then $\mathrm{CH}_{4}$ was supplied into the CVD chamber at a flow rate of $70 \mathrm{sccm}$. When the pressure was increased to $\sim 2 \times 10^{3} \mathrm{~Pa}$ and became stable, the filaments were heated and the substrate temperature was raised to $\sim 930{ }^{\circ} \mathrm{C}$. At this temperature, the CNFs were grown for several minutes. The growth conditions are listed in Table 1.

The structure and composition of synthesized nanomaterials were studied using S-4800 field emission scanning electron microscopy (FESEM) at $15 \mathrm{KV}$, JEOL 2010F high-resolution transmission electron microscopy (TEM) at $200 \mathrm{KV}$, HR 800 micro-Raman spectroscopy using $532 \mathrm{~nm}$ line of semiconductor laser as the excitation source, and ESCALAB 250 X-ray photoelectron spectroscopy (XPS) using $\mathrm{Al} \mathrm{K \alpha} \mathrm{X}$-ray source. The PL properties of the synthesized nanomaterials were investigated at room temperature in a Horiba Scientific Labram HR evolution Raman spectrometer under the excitation of $325 \mathrm{~nm}$ line of He-Cd laser. During the PL measurement, the laser power was $6.8 \mathrm{~mW}$ and the diameter of irradiation spot was $\sim 1$ $\mu \mathrm{m}$. 


\section{Results and discussion}
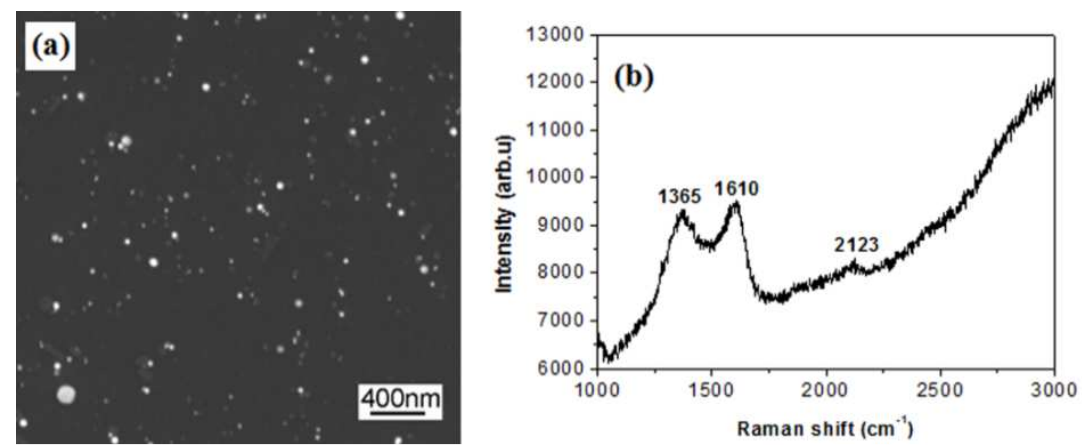

Fig. 2 (a) FESEM image and (b) Raman spectrum of carbon nanodots, which indicates that the size of carbon nanodots is diversified and the carbon nanodots have amorphous structure.

\subsection{Structure and composition of samples}

Figure 2 shows a FESEM image and Raman spectrum of synthesized carbon nanodots. As seen in Fig. 2(a), the sizes of carbon nanodots are diversified, with the typical carbon nanodots having the size in a range of 18 71 $\mathrm{nm}$ and some large nanodots reaching $130 \mathrm{~nm}$. The Raman spectrum of carbon nanodots shows three peaks at about 1365, 1610, and $2123 \mathrm{~cm}^{-1}$, respectively. The peaks at $\sim 1365$ and $1610 \mathrm{~cm}^{-1}$ are the $\mathrm{D}$ and $\mathrm{G}$ peaks of amorphous carbon, and the peak at about $2123 \mathrm{~cm}^{-1}$ is related to the $\mathrm{C}-\mathrm{N}$ bonds $[25,26]$.
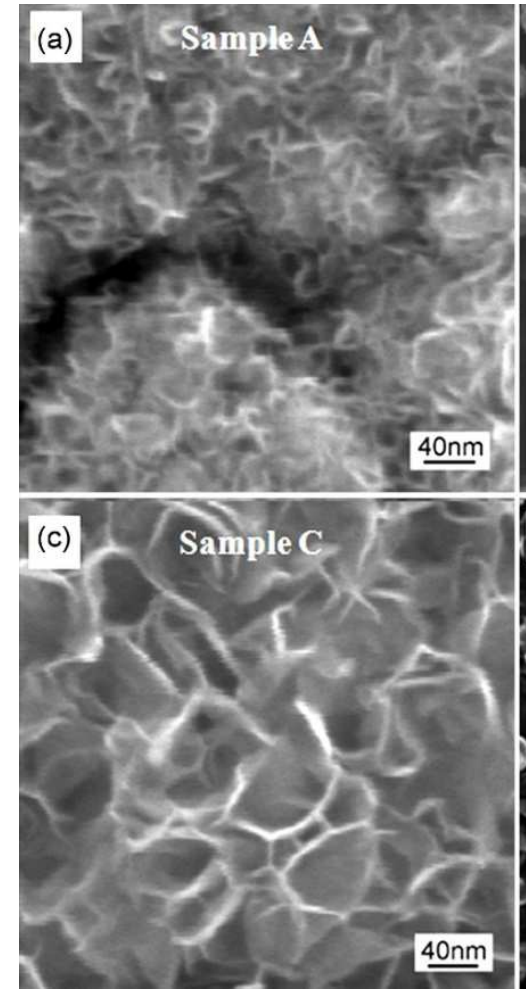
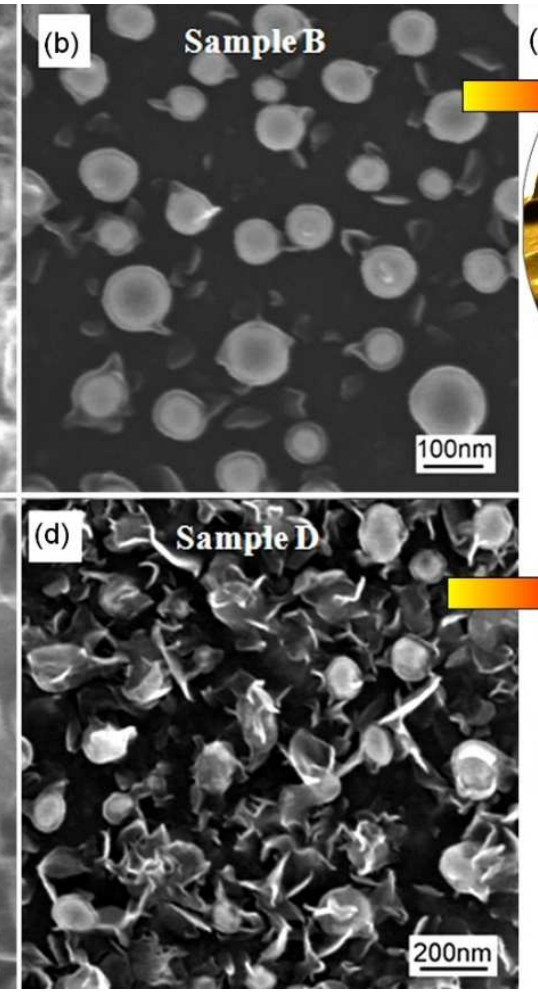

(e)

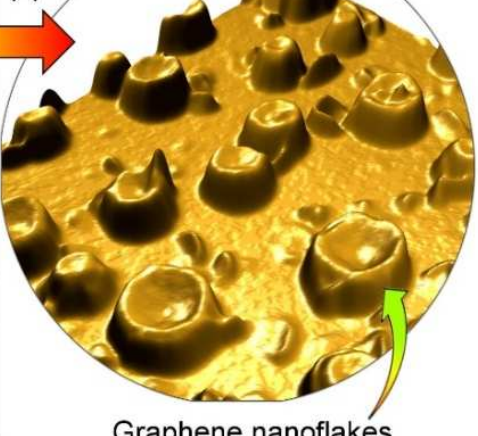

Graphene nanoflakés

(f) around nanoparticles

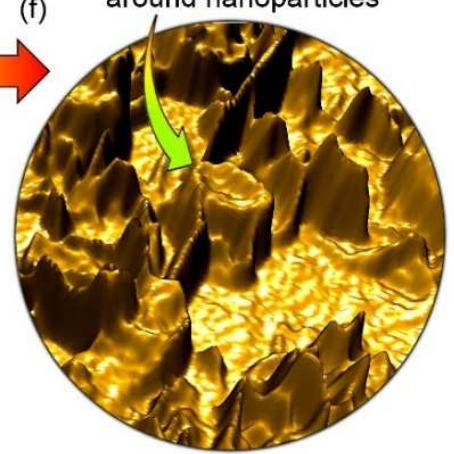

Fig. 3 (a-d) FESEM images of samples A-D, showing that CNFs can nucleate on carbon dots and Au nanoparticles. (e, f) - 3D reconstruction of the structures shown in (b) and (d), respectively. Carbon nanoflakes around the nanoparticles are clearly visible. 


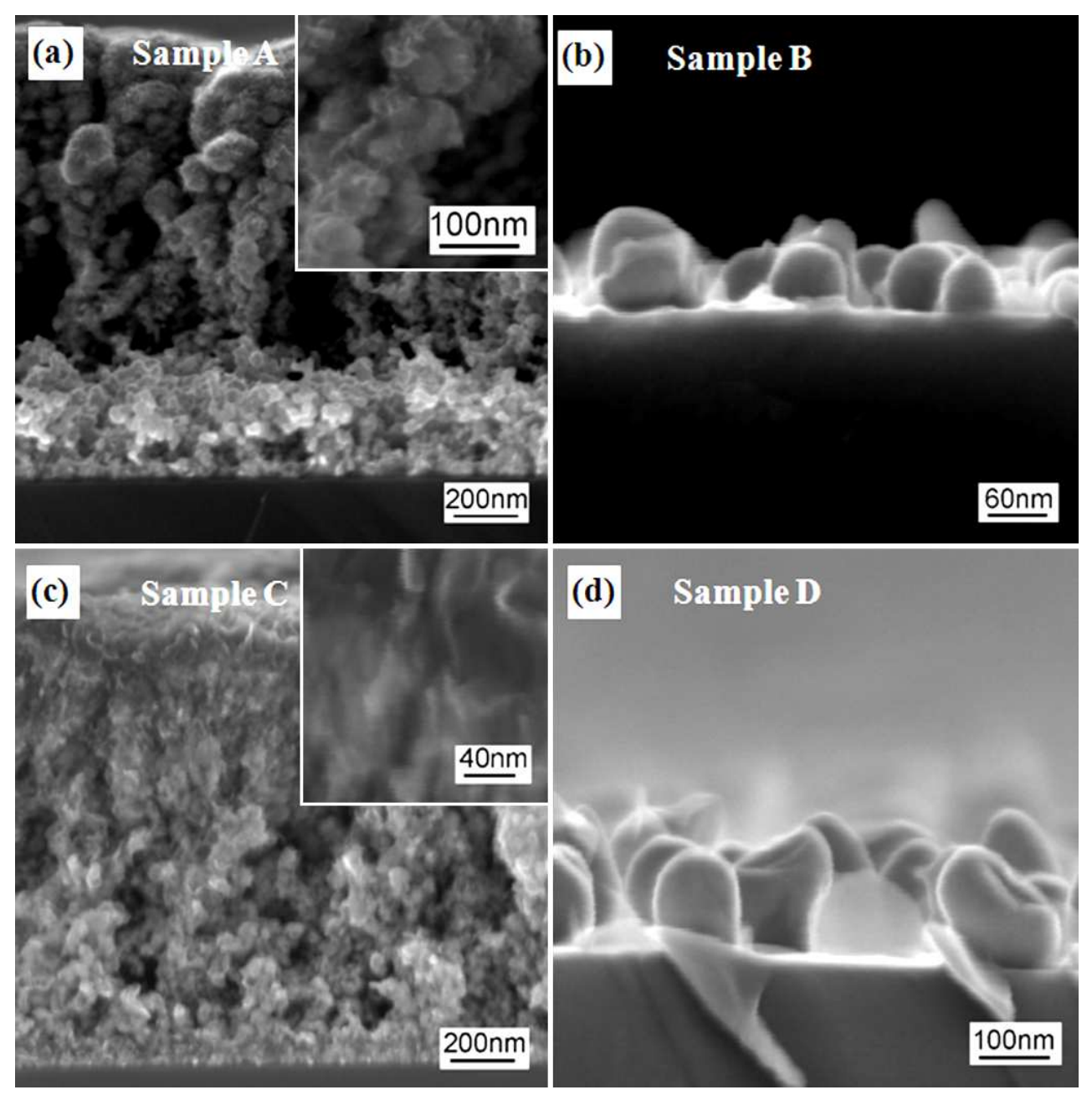

Fig. 4 Cross-section FESEM images of samples A-D. The insets in (a) and (c) are the amplified FESEM images of samples $A$ and $C$. These images indicate that the CNFs on carbon dots have a higher growth rate than the GNFs on Au nanoparticles.

Figures 3 and 4 are the surface and cross-section FESEM images of all samples A-D, respectively. In Fig. 4, the insets are the magnified FESEM images of agglomerates found in samples $\mathrm{A}$ and $\mathrm{C}$, indicating that these agglomerates are composed of nanoflakes. From Fig. 3 and 4 one can apparently see that the nanoflakes nucleated directly on carbon and Au NPs and they grow in an oriented mode. Moreover, Fig. 4 shows a larger nanoflake thickness in samples $\mathrm{A}$ and $\mathrm{C}$ than in $\mathrm{B}$ and $\mathrm{D}$, thus indicating that the nanoflakes in samples $\mathrm{A}$ and $\mathrm{C}$ have a higher growth rate compared to samples B and D.

Figure 5 shows the Raman spectra of samples A-D. Each spectrum in this figure features the $\mathrm{D}, \mathrm{G}, \mathrm{D}$ ', 2D, and $\mathrm{D}+\mathrm{G}$ peaks, and the positions of these peaks are listed in Table 2. Among these peaks, the $D, G, 2 D$, and $D+G$ peaks are quite consistent with the characteristic Raman peaks of graphene flakes reported elsewhere $[1,6,26,27]$, indicating that the synthesized nanomaterials are the graphite carbon nanoflakes. 

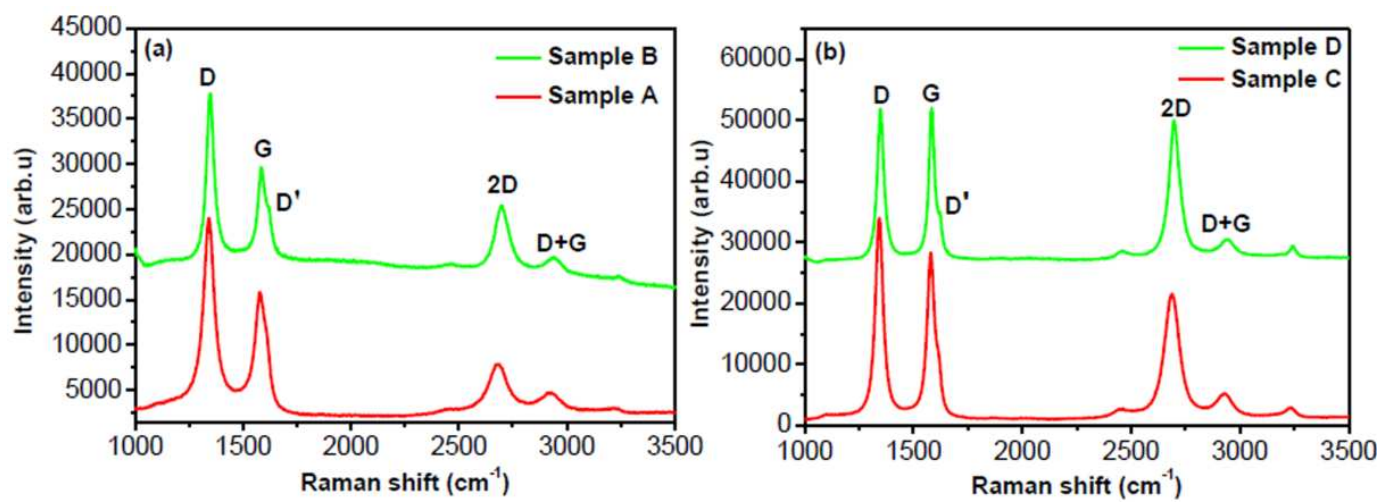

Fig. 5. Raman spectra of samples A-D. Each spectrum shows the $D, G, D^{\prime}, 2 D$, and $D+G$ peaks intrinsic to the graphene structure.

Table 2. Positions of D, G, D', 2D and D+G peaks in Fig.5.

\begin{tabular}{cccccc}
\hline Sample & $\mathbf{D}\left(\mathrm{cm}^{-1}\right)$ & $\mathbf{G}\left(\mathrm{cm}^{-1}\right)$ & $\mathbf{D}^{\prime}\left(\mathrm{cm}^{-1}\right)$ & 2D $\left(\mathrm{cm}^{-1}\right)$ & $\mathbf{D}+\mathbf{G}\left(\mathrm{cm}^{-1}\right)$ \\
\hline A & 1342 & 1579 & 1618 & 2680 & 2929 \\
B & 1349 & 1586 & 1621 & 2700 & 2941 \\
C & 1345 & 1580 & 1619 & 2687 & 2930 \\
D & 1349 & 1584 & 1620 & 2696 & 2940 \\
\hline
\end{tabular}

The origination of $\mathrm{D}^{\prime}$ peak shown in Fig. 5 is attributed to the intravalley resonance scattering caused by the disorder in graphene [28,29], which is related to the defects and doping in graphene [30]. From the XPS results (see Table 3) it follows that there is no component of nitrogen in samples $\mathrm{A}$ and $\mathrm{B}$, i.e., nitrogen was not doped into the CNFs. Thus, the D' peak results from the defects the CNFs. Furthermore, the Raman spectra show strong D peak, which is related to the edge effect of CNFs. Figures 3 and 4 show that the samples are composed of CNFs contianing many edges, while both defects and edges of graphene contribute to the D peak [31,32]; thus, the strong D peak results from the edge effect of CNFs.

To study a fine structure and composition of CNFs, samples A and B were examined using XPS, and samples C and D were investigated using a high-resolution TEM, respectively. Fig. 6 is the high-resolution TEM images of samples C and D, which indicate that the GNFs have a crystalline structure with some amorphous phases (see the areas circulated by red dot lines). We used the standard TEM analytic software to measure the distances between two layers for sample $\mathrm{C}$ and 10 layers for sample D, and the results of measurements are also shown in Fig. 6. 

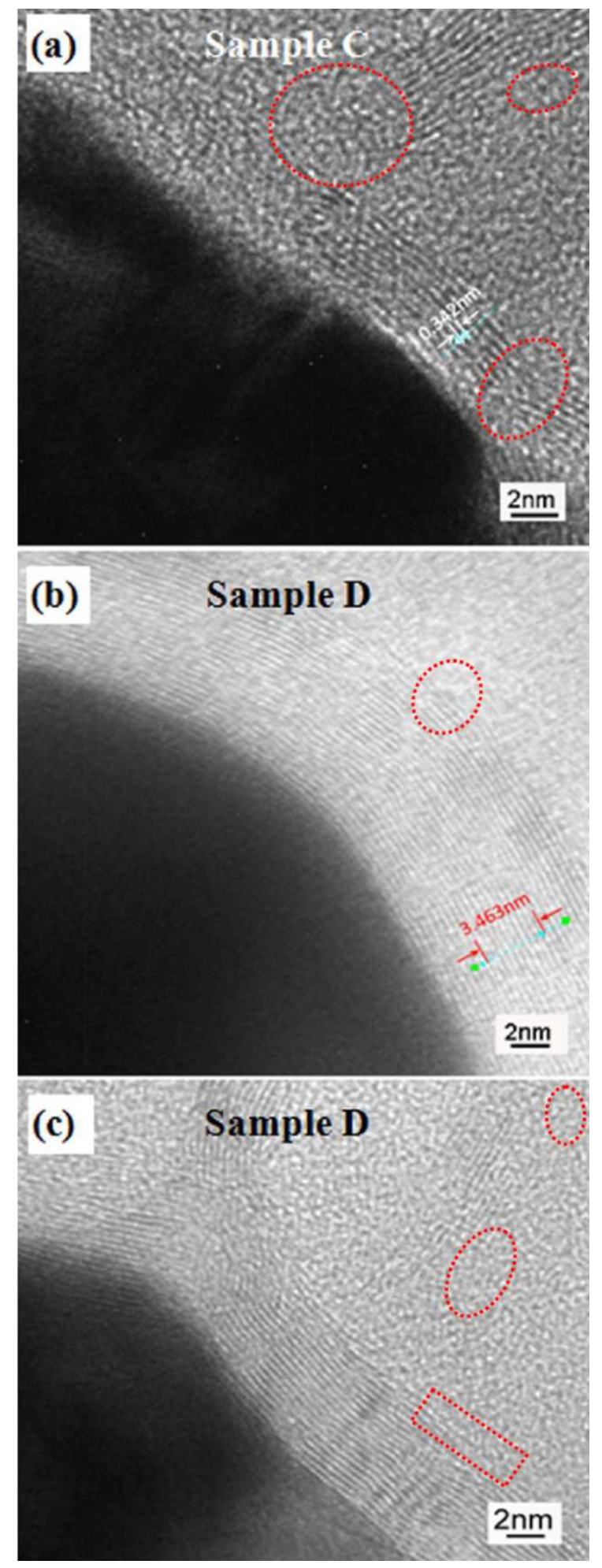

Fig. 6 TEM images of samples $\mathrm{C}$ and D. CNFs have a structure of carbon sheets and some new nanoflakes grow from the graphite carbon covering carbon and gold nanoparticles. The areas circulated by red dot lines are the amorphous phases.

According to these data, the space between two GNF sheets is about $0.34 \mathrm{~nm}$, which is consistent with the space separation between two graphene layers reported in Ref. (18). Furthermore, Fig. 6 shows that the carbon and Au NPs are covered with the graphite carbon layers and some new 
nanoflakes grow from the graphite carbon layers on carbon and Au NPs. From Fig. 6 one can see that the graphite carbon layers are firstly formed on the surfaces of carbon and Au NPs, and then the CNFs are grown from the graphite carbon layers on the carbon and Au NPs in oriented mode.

Figure 7 is the XPS spectra of samples A and B, which show the $\mathrm{C} 1 \mathrm{~s}$ and $\mathrm{O} 1 \mathrm{~s}$ peaks at about 284.8 and $533.5 \mathrm{eV}$. In addition, the spectrum of sample B shows the Au 4f, Si 2p and Si 2s XPS peaks at about 84.5, 103.5 and $153.5 \mathrm{eV}$, respectively [33]. The atomic densities of elements obtained from the XPS results are listed in Table 3. Importantly, there is no nitrogen in sample A, i.e., nitrogen is not doped into the CNFs. While carbon nanodots contain nitrogen (see Fig. S2 in the Supporting Information available), the CNFs were grown in $\mathrm{CH}_{4}$ environment and hence, it is difficult for nitrogen to diffuse from carbon nanodots to CNFs due to fast growth of nanoflakes. No $\mathrm{N}_{2}$ was employed during the growth of samples $\mathrm{B}$, thus there was no nitrogen component in sample B.

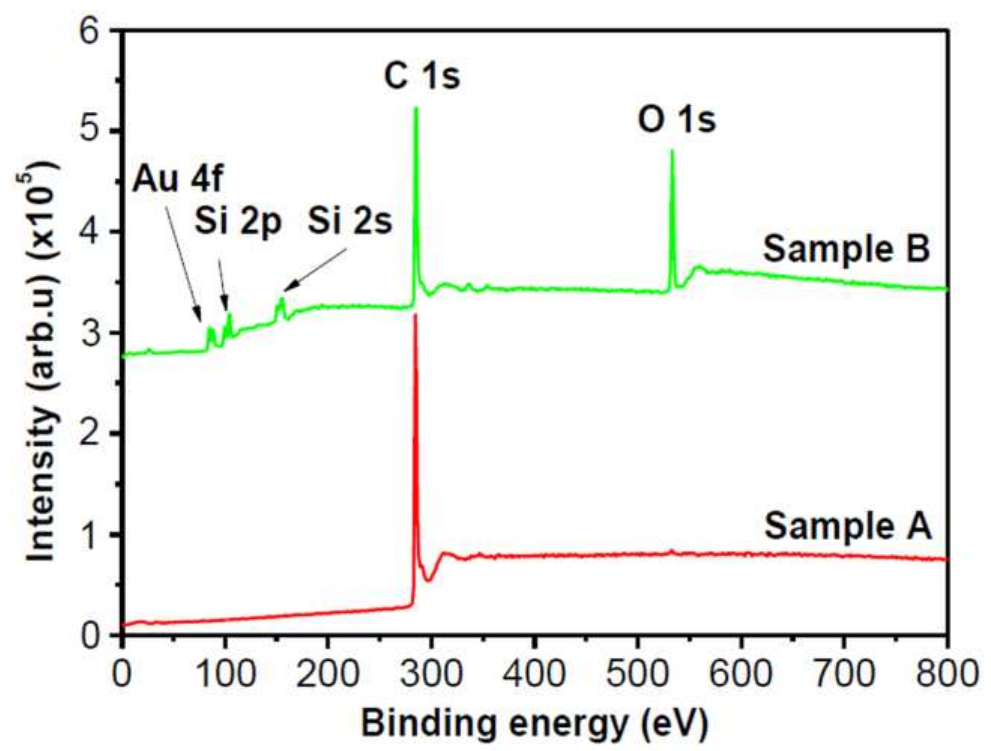

Fig. 7. XPS spectra of sample A and B. The Au 4f, Si 2p, Si 2s, C 1s and O 1s XPS peaks are present at about 84.5, $103.5,153.5,248.8$ and $533.5 \mathrm{eV}$, respectively.

Table 3 Atomic density of elements obtained from XPS measurement.

\begin{tabular}{ccccc}
\hline Sample & C (at.\%) & O (at.\%) & Au (at.\%) & Si (at.\%) \\
\hline A & 99.17 & 0.83 & - & - \\
B & 67.67 & 15.55 & 0.65 & 16.14 \\
\hline
\end{tabular}




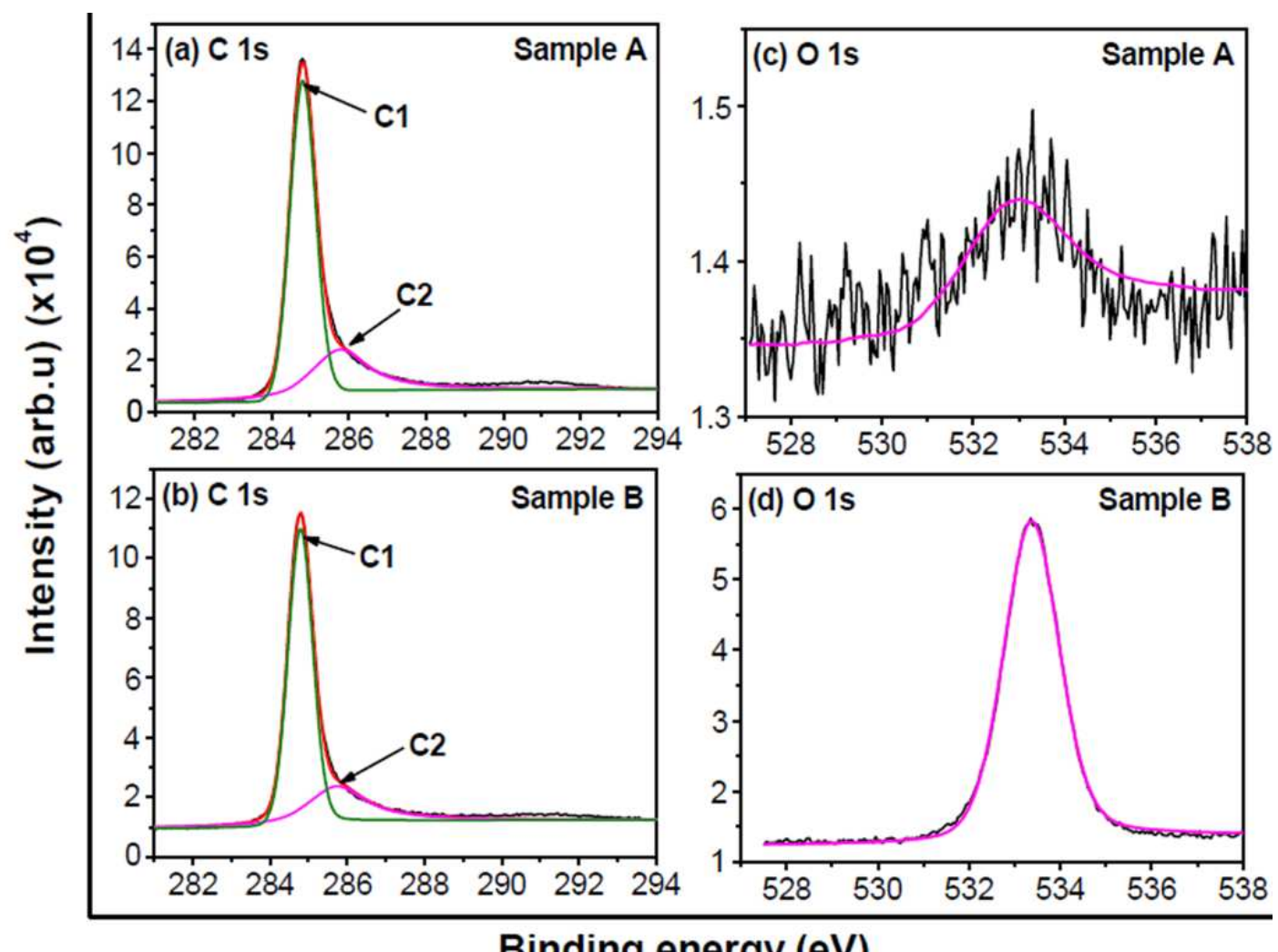

Binding energy (eV)

Fig. 8. Amplified C 1s and O 1s XPS spectra of samples A and B. The C1 and C2 peaks are fitted, which indicate that the graphite carbon nanoflakes contain $s p^{3}$ carbon.

Table 4. The positions of fitted XPS peaks shown in Fig. 8

\begin{tabular}{cccc}
\hline Sample & C1 (eV) & C2 (eV) & O1 (eV) \\
\hline A & 284.79 & 285.80 & 533.1 \\
B & 284.79 & 285.77 & 533.3 \\
\hline
\end{tabular}

To further confirm the binding states of $\mathrm{C}$ and $\mathrm{O}$ elements, the $\mathrm{C} 1 \mathrm{~s}$ and $\mathrm{O} 1 \mathrm{~s}$ XPS peaks were fitted using the standard XPS fitting software after Shirley background subtraction. The fitted peaks are shown in Fig. 8 and their positions are listed in Table 4. The C1 and C2 peaks shown in Fig. 8 are attributed to the $s p^{2}$ and $s p^{3} \mathrm{C}-\mathrm{C}$ bonds, respectively [1,34]. The fitted $\mathrm{O} 1 \mathrm{~s}$ peaks are located at $\sim 533 \mathrm{eV}$ and relate to the $\mathrm{C}-\mathrm{O}$ or $\mathrm{Si}-\mathrm{O}$ bonds, respectively [33]. In Fig.7, the O 1s XPS peak of sample B is much stronger than that of sample A, which may result from the native $\mathrm{SiO}_{2}$ on the silicon substrate surface. From Fig. 7 one can see that the two XPS peaks related to $\mathrm{Si}$ are located at about 103.5 and $153.5 \mathrm{eV}$, apparently they result from $\mathrm{Si}$ element in $\mathrm{SiO}_{2}$ [33]. Fig. 4 shows that the CNF film of sample $\mathrm{B}$ is much thinner than sample A, which implies that the surface of Si substrate is easily detected by XPS; in other words, O element in 
$\mathrm{SiO}_{2}$ on the $\mathrm{Si}$ substrate is detected by XPS, thus the XPS spectrum of sample B shows a very strong peak related to oxygen.

\subsection{Formation and growth of carbon nanoflakes on carbon and Au nanoparticles}

The obtained results indicate that the graphite carbon layers firstly nucleate on surfaces of carbon and Au NPs, and then the oriented CNFs start growing. Importantly, the growth of oriented CNFs on the carbon NPs is faster than the growth on the Au NPs. In this section, the formation and oriented growth of CNFs are analyzed.

During the growth of CNFs, $\mathrm{CH}_{4}$ gas was supplied into the CVD chamber before heating, i.e., $\mathrm{CH}_{4}$ molecules could absorb on the surfaces of carbon NPs and Au film. During heating of the filament and substrates, $\mathrm{CH}_{4}$ molecules are decomposed into hydrocarbon chemicals and atomic hydrogen:

$$
\mathrm{CH}_{4} \rightarrow \mathrm{CH}_{3}+\mathrm{H} \text {. }
$$

Atomic hydrogen has a high chemical activity, so it easily reacts with $\mathrm{CH}_{4}$ molecules absorbed on the carbon NPs and Au film and forms carbon atoms through the dehydrogenation reaction. Finally, these atoms form the graphite carbon layers on carbon NPs and Au NPs formed due to the melting of continuous Au film $[19,26,35]$. However, there are a great difference in the formation rate of graphite carbon layers on carbon and Au NPs. When the substrates are heated, the carbon NPs can promote decomposition of hydrocarbon chemicals because carbon black has a catalytic effect towards decomposition of $\mathrm{CH}_{4}$ at high temperature [36]. As a result, the formation rate of graphite carbon layers is high on the carbon NPs. Besides, carbon nanodots can be evaporated during the formation of graphite carbon layers, which contribute to the growth of graphite carbon layers. However, the TEM image shown in Fig. 6(a) indicates that the graphite carbon nanoflakes are rested on carbon nanodots, i.e., the carbon nanodots survive after the nucleation and growth of nanoflakes. Due to the difference in sizes of carbon nanodot shown in Fig. 2 it is possible that large carbon nanodots serve as a source of carbon for CNF growth, and some small carbon nanodots can be completely consumed during the formation of CNFs. In other words, the consumption of carbon nanodots is a factor enhancing the growth rate of CNFs. On the other hand, the surfaces of carbon nanodots are fast covered by hydrocarbon radicals due to a high flow rate of $\mathrm{CH}_{4}$, which decreases the evaporation rate of carbon nanodots. Therefore, 
the reaction of hydrocarbon radicals on the surfaces of carbon nanodots mainly contributes to the growth of graphene nanoflakes. In case of Au NPs, the inertness of Au results in the desorption of $\mathrm{CH}_{4}$ molecules from the surfaces of Au NPs during heating, i.e., the formation rate of carbon atoms is slow before $\mathrm{Au}$ NPs are not molten. When $\mathrm{Au}$ NPs melt, the absorption of $\mathrm{CH}_{4}$ molecules improves the dissolution of carbon atoms in the molten Au NPs [37,38]. Due to the Gibbs-Thomson effect, the dissolved carbon atoms precipitate from the Au NPs and form the graphite carbon layers on the surfaces of Au NPs [39]. The Gibbs-Thomson effect here refers to the precipitation of dissolved atoms in catalyst particles, driven by the high stress/inner pressure difference formed due to the size and solubility of catalyst particles [40,41]. Thus, it takes a long time to form the graphite carbon layers on the Au NPs, which means that the formation rate of graphite carbon layers on the Au NPs is slow.

During the formation of graphite carbon layers, the amorphous carbon and Au NPs produce deformation [42-44], which results in the production of stress in the graphite carbon layers. Furthermore, the difference in the thermal expansion rates of graphite carbon layers with carbon and $\mathrm{Au}$ NPs further increase the stress in the graphite carbon layers [39]. As a consequence, some carbon sheets in the graphite carbon layers can be broken to form the cracks at the sites with high stress. Finally, the edges of cracks bend upward under the stress to lead to the formation of oriented carbon nanoflakes (i.e., oriented CNFs) [22,45].

In the previous works, the oriented growth of graphene by PECVD was considered to result from the change of growth direction of graphite layers caused by the stress, and the oriented growth of graphene was controlled by the electric field produced by plasma [22,23]. In our work, the growth of CNFs was carried out in HFCVD system, where there is no plasma to form the electric field. The oriented CNFs growth in HFCVD system may be related to the charge transfer in the hydrocarbon radicals. According to Eq. (1), a number of hydrocarbon radicals are formed during the growth of CNFs. The difference in the electronegative values of $\mathrm{H}$ and $\mathrm{C}$ elements results in the charge transfer between $\mathrm{H}$ and $\mathrm{C}$ atoms. As a result, a hydrocarbon radical can acquire a net charge. When the hydrocarbon radicals deposit onto the edges of bending cracks, the edges of cracks possess net charge, which leads to a repulsion effect between two cracks. The repulsion effect between two cracks maintains the orientation growth of CNFs.

Due to the high formation rate of graphite carbon layers on the carbon NPs relative to Au 

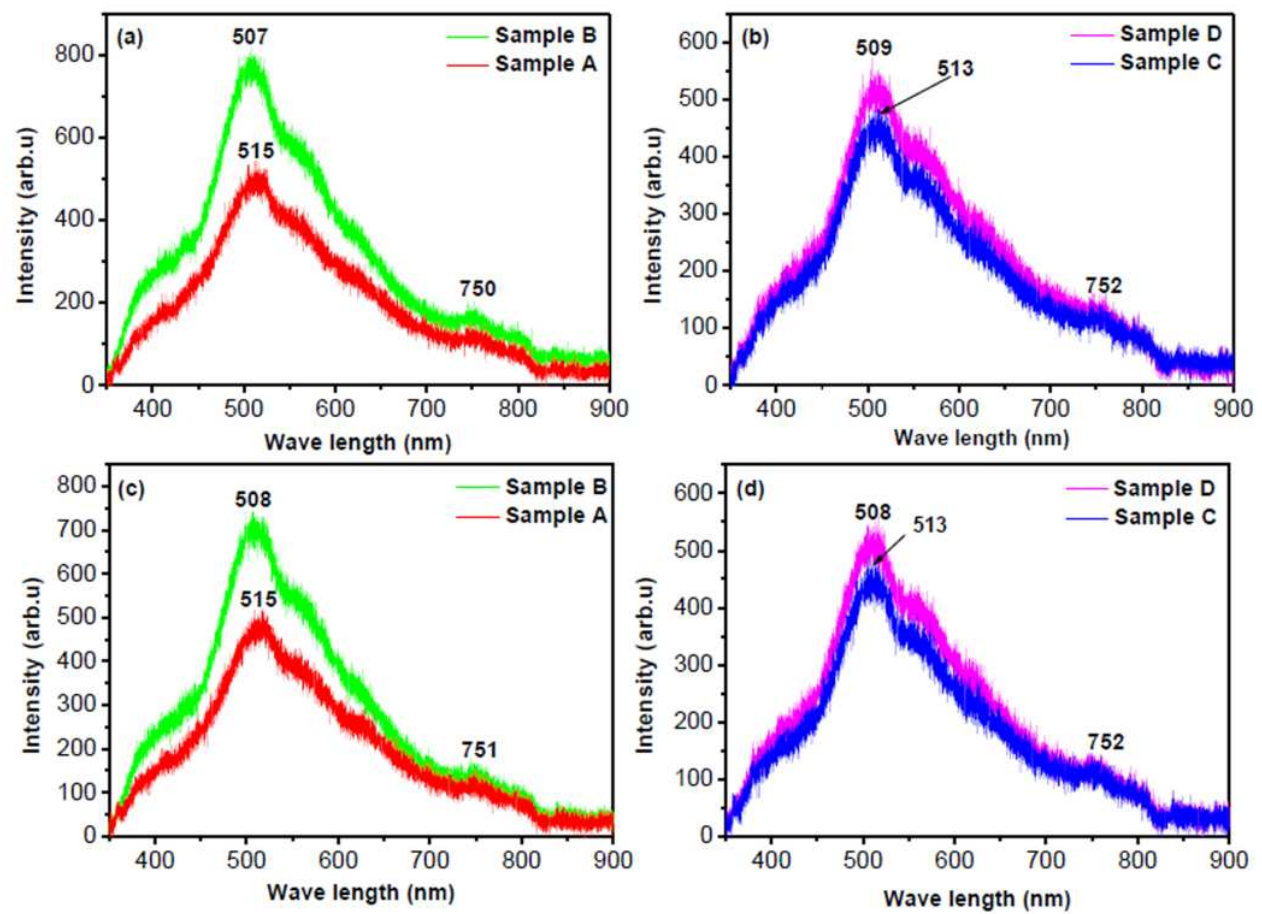

Fig. 9 PL spectra of samples A-D, which show the green and red PL bands at about 507-515 and 750-752 nm, respectively. Stronger PL intensity of samples $B$ and $D$ than $A$ and $C$ indicates that the PL emission is enhanced by Au nanoparticles.
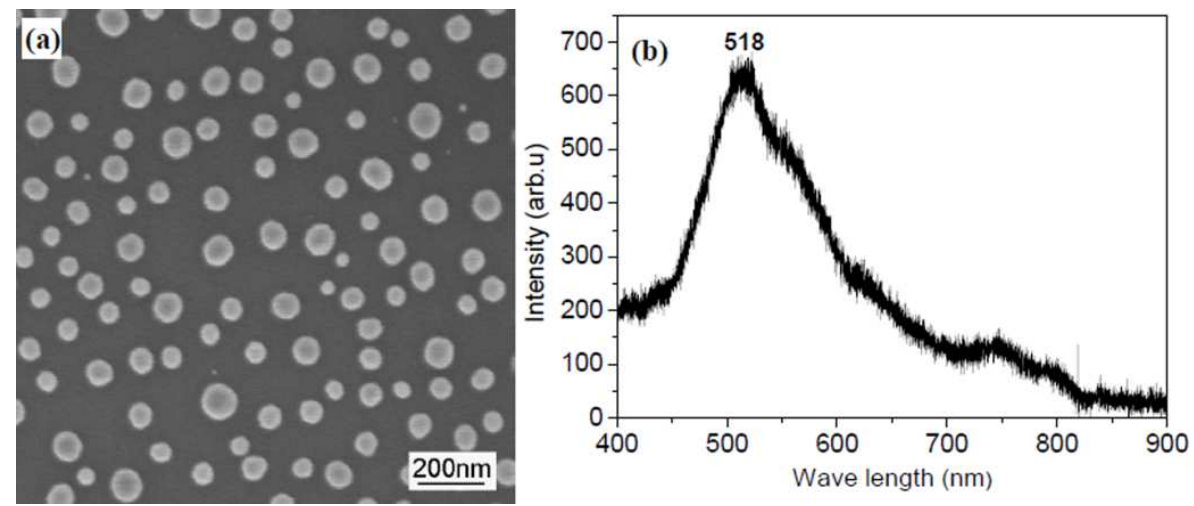

Fig. 10. (a) SEM image and (b) PL spectrum of molten Au NPs. The Au NPs was formed in $\mathrm{H}_{2}$ environment for 1 min at $850-900{ }^{\circ} \mathrm{C}$.

NPs, the rate of crack formation on the carbon NPs is higher than the Au nanoparticles. As a result, Fig. 3 shows more CNFs for samples A and C than for samples B and D, and Fig. 4 exhibits a larger thickness of CNF films of samples A and C compared to samples B and D.

\subsection{PL properties of carbon nanoflakes on carbon and Au nanoparticles}

Figure 9 is the PL spectra obtained from different area of samples A-D, which confirm that relative intense PL emission generates from the synthesized nanostructures. From Fig. 9 one 
apparently can see that the samples generate the green and red PL bands centered at about 507-515 and 750-752 nm, and the PL intensity of samples B and D are stronger than that of samples A and C, respectively. In addition, the PL spectra show the broad PL bands and low intensity. Since Au NPs can emit green PL band [46,47], we have measured the PL spectrum of $\mathrm{Au}$ NPs and presented the results in Fig. 10. From this graph one can see that the Au NPs generate the PL band at about $518 \mathrm{~nm}$, i.e., the PL bands (507-509 nm) of samples B and D are not the PL bands intrinsic to Au NPs. Fig. 4 shows a much thicker CNF structures on samples A and $\mathrm{C}$ than on samples $\mathrm{B}$ and $\mathrm{D}$, which implies that the PL emission from samples A and C was indeed generated by CNFs. Thus, strong PL intensity of samples B and D compared to samples A and $\mathrm{C}$ indicates that the PL emission from CNFs was enhanced by the Au nanoparticles, and the enhancement of the PL emission relates to the CNF-Au interfaces. In the next section the PL properties of CNFs on carbon and Au NPs are discussed.

\subsubsection{PL generation of PL bands of CNFs}

It is known that graphene is a carbon material with zero bandgap, i.e., graphene cannot generate PL emission. Thus, how do the CNFs generate PL emission? To study the PL origination in CNFs, the composition of CNFs was studied in details using Fourier transform infrared (FTIR) spectroscope, and the FTIR spectrum is shown in Fig. S1 in the Supporting Information. The spectrum indicates that the CNFs contain $\mathrm{C}-\mathrm{H}$ and $\mathrm{C}-\mathrm{O}$ radicals, which are related to the residual oxygen and hydrocarbon radicals present in CVD chamber. However, the C-H radicals such as aromatic and olefinic molecules are usually responsible for the blue PL emission [48], while the C-O radicals relate to red PL emission [49], and green PL emission is related to the $s p^{2}$ clusters [6,50]. From Fig. 9 it is seen that the CNFs generate strong green PL bands, which implies that the PL properties of CNFs are related to the carbon clusters. The PL emission of carbon nanodots shown in Fig. 2 were further measured at the same conditions as samples A-D.

Figure 11 is the PL spectra obtained from different areas on carbon nanodot sample. As shown in this graph, the carbon nanodots generate the PL bands at about 392, 506, 510 and 750 $\mathrm{nm}$, which are similar to the PL spectra of amorphous carbon nitride films [51]. In the Ref. (51), Iwano interpreted the PL generation by the transition mechanism, i.e., the transition with energy of $1.4-1.9 \mathrm{eV}$ is attributed to the interband transition between $\pi^{*}$ conduction band and lone-pair 
valence band of nitride, the transition with energy exceeding $2 \mathrm{eV}$ occurs between $\pi^{*}$ conduction band and $\pi$ valence band, or $\sigma^{*}$ conduction band and lone-pair valence band of nitride. The Raman spectrum of carbon nanodots shown in Fig. $2 b$ indicates that the carbon nanodots feature an amorphous structure. From the XPS spectra of carbon nanodots shown in Fig. S2 in supporting information, the carbon nanodots consist of $s p^{2} \mathrm{C}-\mathrm{C}, s p^{2} \mathrm{C}-\mathrm{N}, s p^{3} \mathrm{C}-\mathrm{C}$ and C-O species. According to the PL mechanism of amorphous carbon nitride films, the PL band at about $392 \mathrm{~nm}$ can be attributed to the transition between $\sigma^{*}$ conduction band and lone-pair valence band of nitride, the PL band at about 506-510 $\mathrm{nm}$ originates from the transition between $\pi^{*}$ conduction band and $\pi$ valence band and the PL band at $\sim 750 \mathrm{~nm}$ occurs the transition between $\pi^{*}$ conduction band and the oxygen defect states formed between $\pi^{*}$ conduction band and $\pi$ valence band.

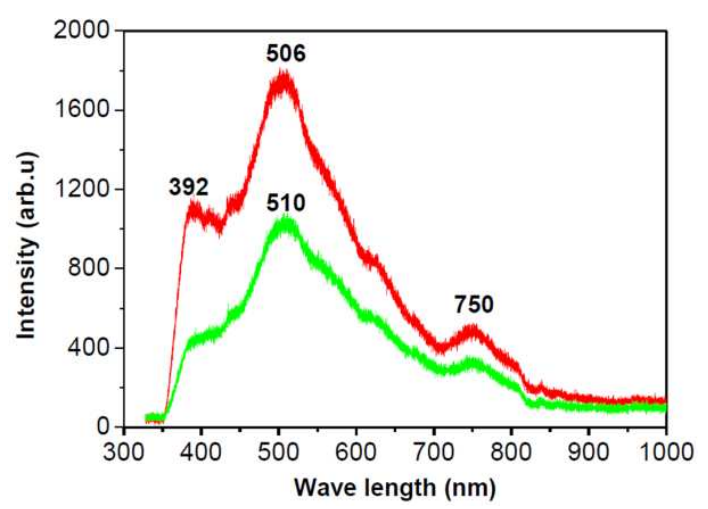

Fig. 11. PL spectra of different areas on carbon nanodot sample, indicating that the carbon nanodots generate blue, green and red PL bands at about 392, 506, 510 and $750 \mathrm{~nm}$, respectively.

The TEM images shown in Fig. 6 indicate that there are amorphous phases in the CNFs and edges of CNFs (see the areas circulated by the dot red lines). In particular, the amorphous phases isolate small $s p^{2}$ carbon clusters on the edges of CNFs. The XPS results indicate the formation of $s p^{3}$ carbon in the CNFs, which is mainly located on the edges of nanoflakes (because the strong D peaks in the Raman spectra relate to the edges of graphene $[31,32])$. As a result, the isolated $s p^{2}$ carbon clusters within $s p^{3}$ carbon lead to the formation of electrons and holes on the $\pi^{*}$ and $\pi$ bands of isolated $s p^{2}$ carbon clusters under ultraviolet excitation [50], and the electron transition between $\pi^{*}$ and $\pi$ bands results in the green PL emission [51]. From XPS spectra, C-O radicals are formed in the CNFs, thus oxygen defect level can form between $\pi^{*}$ and $\pi$ bands of isolated $s p^{2}$ carbon clusters and the electron transition between $\pi^{*}$ and oxygen defect level results in the red PL emission [49]. 
According to the above analyses, the PL generation of CNFs are related to the edges of CNFs. Comparing Fig. 9 with Fig. 11 one can find that the PL spectra of samples B and D are close to the PL spectra of carbon nanodots, thus the PL bands of samples B and D originate from the CNFs. Furthermore, Fig. 6(a) indicates that samples A and C are composed of CNFs and carbon nanodots. From Fig. 4 one can see that CNF are very thick compared to the carbon nanodots; due to the exponential relation of optical decay with distance, the carbon nanodots play a small role for the PL emission of CNFs, and hence, nitrogen in the carbon nanodots have weak effect on the PL emission.

As stated above, the PL generation of CNFs relates to the isolated $s p^{2}$ carbon clusters on the edges of CNFs. The TEM images in Fig. 6 indicate that the sizes of carbon clusters are diversified, and this naturally leads to the broad PL bands since the energy gap of $\pi^{*}$ band and $\pi$ band of a $s p^{2}$ carbon cluster is related to its size [50]. The low PL intensity of CNFs may results from their thickness. The TEM images in Fig. 6 indicate that the CNFs are thick carbon nanosheets, which feature strong absorption of light. As a result, the PL intensity becomes weak. To increase the PL intensity of CNFs, the thickness of CNFs can be reduced to lower the absorption for light. As a result, the PL intensity becomes weak.

To analyze the PL uniformity of samples, we have made a the green PL mapping of samples $\mathrm{C}$ and D, and the results are shown in Fig. 12. According to the color bars, the difference between the strongest and weakest intensities is about $40 \%$ and $20 \%$ for samples C and D, respectively. These data indicate that the photoluminescence of samples grown on $\mathrm{Au}$ nanoparticles has a good uniformity, while the PL uniformity of samples grown on the carbon nanoparticles is relatively poor, which may result from the non-uniformity of CNF thickness.
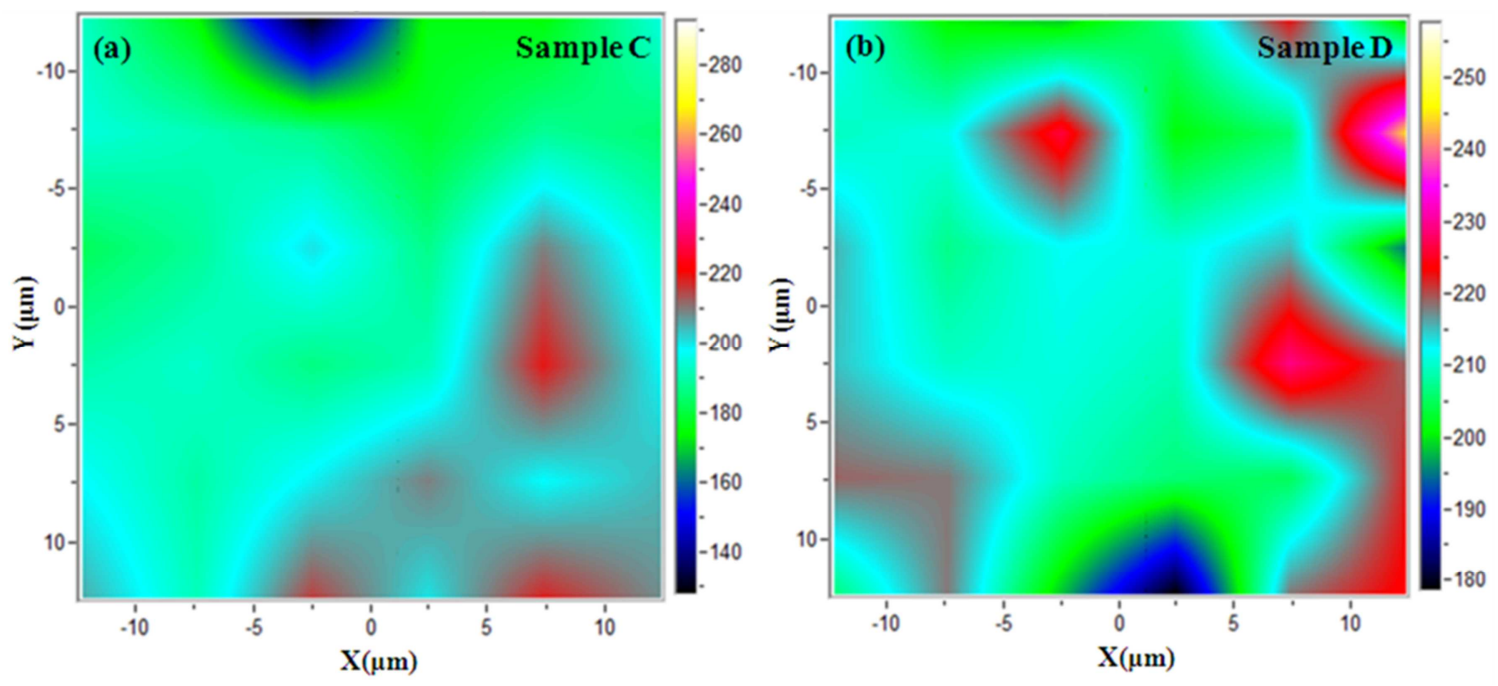

Fig. 12 PL intensity images of samples C and D. The images are obtained from 400-650 nm. 


\subsubsection{PL properties of CNFs}

Figure 9 shows that the PL intensity of sample B is stronger than that of sample A. Similarly, the PL intensity of sample D is stronger than that of sample C; this indicates that the PL emission of CNFs is enhanced Au NPs. In this section we examine the PL enhancement due to processes at the GNF-Au interfaces.

The results described in previous works indicate that the rough Au film or Au nanoparticles can generate the green PL emission under the irradiation of ultraviolet (UV) light due to recombination of electrons near the Fermi level with holes in the $d$ band [46,47] and the observed green PL emission from Au NPs is considered to be the consequence of PL emission enhanced by the subsequent plasmon radiation generated from the process of which the holes in the $d$ band recombine with $s-p$ electrons [52]. In other words, the recombination of electrons near the Fermi level with holes in the $d$ band includes the radiative and non-radiative process, where the radiative combination emits the photons, and the non-radiative combination gives rise to the plasmon emission.

Figures 4 and 6 indicate that in our samples the irregular Au NPs are covered with the carbon layers containing the graphite carbon layers and CNFs, thus light cannot directly irradiate the Au NPs. It is known that the reflection and refraction occur on the interface of two different media when light irradiates the interface between them. Figure 6 shows that the thickness of carbon layers on the Au NPs is inhomogeneous, thus some light can penetrates through thin carbon layers when the sample is irradiated by UV light with a wavelength of $325 \mathrm{~nm}$. As a result, the Au NPs can locally generate the green PL emission and plasmon emission due to the recombination of electrons near the Fermi level with holes in the $d$ band [52]. Due to the local plasmon emission, the local electric field in the vicinity of Au NPs is enhanced, which causes the enhancement in the PL emission from CNFs [53].

Furthermore, plasmon emission can enhance the PL emission from the Au NPs [52]. Due to the carbon layers on gold nanoparticles, the PL emission from the Au NPs must penetrate through the carbon layers, and thus the PL emission of CNFs is further excited and enhanced. However, the plasmon emission from the Au NPs simultaneously results in the transfer of electrons from the Au NPs to graphite carbon layers [54]. As a result, the rate of radiative recombination of electrons near the Fermi level with holes in the $d$ band is reduced to weaken the 
effect of PL emission from the Au NPs on the enhancement of emission from CNFs. It is the possible reason that the PL enhancement of the hybrid of noble metal NPs (e.g., Ag and Au) with graphene or graphene quantum dots is usually interpreted to originate from the surface plasmon of noble metal NPs $[12,15]$.

In addition, the energy transfer simultaneously occurs in the GNF-Au interfaces [17], however the effects of energy transfer on PL emission is related to the distance between the PL emission sites of CNFs and Au NPs, because the surface energy transfer rate $E$ depends on the distance $l$ between donor and acceptor by

$$
E=\frac{N}{N+\left(l / d_{0}\right)^{4}},
$$

where $d_{0}$ and $N$ present the Förster separation and the number of metal NPs interacting with a single donor [55]. It was already demonstrated that $d_{0}$ is in a range of 2-4 $\mathrm{nm}$ and it is rarely larger than $5 \mathrm{~nm}$ [56], thus the PL quenching of fluorophores by energy transfer occurs in a distance below $5 \mathrm{~nm}[56,57]$. With the distance $l$ increasing, the energy transfer rate is greatly reduced according to (2), and this results in the enhancement of photoluminescence due to the enhancement of surface electric field of metal NPs [57].

From Fig. 6 we have also obtained the thickness of graphite carbon layers which are of about 7-10 nm, thus the quenching and enhancement of PL emission simultaneously occur all the graphite carbon layers. In other words, the net effects of charge transfer depend on the thickness of graphite carbon layers on the Au NPs. The 7-10 nm graphite carbon layers on the Au NPs imply that the net effects of charge transfer on the PL emission should result in the PL quenching, however the PL enhancement by the plasmon emission may exceed the PL quenching caused by the charge transfer. It is the possible reason that our results exhibit the PL enhancement on CNFs interfaced with gold nanoparticles.

Compared the PL spectra of samples A and B with the spectra of samples C and D, the PL enhancement of sample D relative to sample C is weaker than that of sample B relative to the sample A. To confirm the PL properties of samples C and D, the PL emission of samples C and D was measured for many times. The mult-measurements exhibit similar results (see Fig. 9 and Fig. S3 in supporting information), which indicate that the PL intensity of sample D is stronger than that of sample C. Thus, the PL emission of CNFs was indeed enhanced by Au nanoparticles, but the PL improvement of sample $\mathrm{D}$ relative to sample $\mathrm{C}$ is not strong, and this may be related 
to the increase of CNF area. The area of CNFs increased due to the long growth time for samples $\mathrm{C}$ and D, as confirmed by Fig. 3; this leads to the increase of number of defects in CNFs including the defects between carbon sheets (due to multilayer structure) and the amorphous phases in the interior of CNFs. Thus, the number of dangling bonds in the CNFs (i.e., the number of PL quenching centers in the CNFs) increased. As a result, the PL enhancement of sample B relative to sample $\mathrm{A}$ is stronger than that of sample $\mathrm{D}$ relative to sample $\mathrm{C}$. In other words, the effect of Au NPs on the PL enhancement of CNFs weakens with the increase of CNF area.

\subsection{Potential applications of CNFs for biology studies}

Figures 3 and 4 show that the CNFs have vertical orientation and open structure. Due to a unique structure of CNFs, the analytes can fully access the surfaces and edges of CNFs like in the case of vertical graphenes [22], thus such CNFs can be used as a platform for the advanced biosensors. In particular, Fig. 6 indicates that the surfaces and edges of CNFs contain some micropits. When the CNFs are placed in the solution containing biological macromolecules such as proteins, the proteins are easily absorbed in the micropits due to a pitted structure. After they are irradiated by light, the energy transfer between the proteins and CNFs can result in changes of shape and spectral position of PL bands with the excitation wavelength, like PL properties of proteins on nanodiamond [58]. Similarly, the CNFs can be used for biocompatible labels [58]. Furthermore, the CNFs easily capture and stabilize the biomedical labeling reagents (i.e., highly luminescent semiconductor nanoparticls) in solution, which are similar to the capture and stabilization of CdSe nanoparticles by dendron ligands [59]. After they are excited by light, the carbon walls and edges will be labelled like the label of sidewalls of carbon nanotubes by semiconductor quantum dots; thus, the CNFs can be used to fabricate the biological nanoprobes for cytological studies [60]. The above applications of CNFs in biology science are our subject of future work.

\section{Conclusion}

In summary, the oriented carbon nanoflakes were grown on amorphous carbon and gold NPs in $\mathrm{CH}_{4}$ environment by HFCVD, and their structure and composition were studied by various characterization techniques including FESEM, high-resolution TEM, Raman and XPS. The 
oriented growth mechanism and room temperature PL properties of CNFs were studied with relation to the processes at the CNF-carbon NP and CNF-Au interfaces. The formation of oriented CNFs originates from (1) the stress in the graphite carbon layers on carbon and Au NPs due to the deformation of carbon and Au NPs and (2) the difference in the thermal expansion rates of graphite carbon layers with carbon and Au NPs. The oriented growth of CNFs is maintained by the repulsion between two CNFs caused by the net charge produced from the hydrocarbon radicals on the edges of CNFs due to the charge transfer between $\mathrm{H}$ and $\mathrm{C}$ atoms. The PL generation of CNFs is related to the edges of CNFs, which results from the $s p^{2}$ carbon clusters on the edges of CNFs. The strong PL emission of CNFs on Au NPs compared to the CNFs on carbon NPs results from the plasmon emission of Au NPs generated from the non-radiative recombination of electrons near the Fermi level with holes in the $d$ band of Au NPs under irradiation of UV light. These achievements can be used to control the structure of hybrid graphene nanomaterials [61,62], metamaterials [63], enhance optical properties of graphene-based nanomaterials, and contribute to the development of optoelectronic devices of graphene-based nanomaterials grown in complex plasmas [64].

\section{Acknowledgements}

This work was partially supported by CSIRO's OCE Science Leadership Scheme and the Australian Research Council. I. L. acknowledges the support from the School of Chemistry, Physics and Mechanical Engineering, Science and Engineering Faculty, Queensland University of Technology. We would like to appreciate Prof. Y. Yang of Institute of Physics of Chinese Academy of Science for the discussion on the PL uniformity of samples.

\section{References}

[1] D. H. Seo, S. Pineda, J. Fang, Y. Gozukara, S. Yick, A. Bendavid, S. K. H. Lam, A. T. Murdock, A. B. Murphy, Z. J. Han. K. Ostrikov, Single-step ambient-air synthesis of graphene from renewable precursors as electrochemical genosensor, Nat. Commun. 8 (2017) 14217-9.

[2] I. Levchenko, K. K. Ostrikov, J. Zheng, X. Li, M. Keidar, K. B. K. Teo, Scalable graphene production: 
perspectives and challenges of plasma applications, Nanoscale 8 (2016) 10511-10527.

[3] Y. Zhao, Q. Zhang, K. Chen, H. Gao, H. Qi, X. Shi, Y. Han, J. Wei, C. Zhang, Triphenothiazinyl triazacoronenes: donor-acceptor molecular graphene exhibiting multiple fluorescence and electrogenerated chemiluminescence emissions, J. Mater. Chem. C, (2017, in press, DOI: $10.1039 / \mathrm{c} 7 \mathrm{tc00314e)}$.

[4] F. Shayeganfar, R. Shahsavari, Oxygen- and lithium-doped hybrid boron-nitride/carbon networks for hydrogen storage, Langmuir 32 (2016) 13313-13321.

[5] S. Y. Choi, S. H. Baek, S.-J. Chang, Y. Song, R. Rafique, K. T. Lee, T.J. Park, Synthesis of upconversion nanoparticles conjugated with graphene oxide quantum dots and their use against cancer cell imaging and photodynamic therapy, Biosensors and Bioelectronics 93 (2017) 267-273.

[6] B. Wang, K. Ostrikov, T. van der Laan, R. Shao, L. Li, Structure and photoluminescence of boron-doped carbon nanoflakes grown by hot filament chemical vapour deposition, J. Mater. Chem. C 3 (2015) 1106-1112.

[7] Y. Wang, B. Chen, D. H. Seo, Z. J. Han, J. I. Wong, K. Ostrikov, H. Zhang, H. Y. Yang, MoS 2 -coated vertical graphene nanosheet for high-performance rechargeable lithium-ion batteries and hydrogen production, NPG Asia Materials, NPG Asia Mater. 8 (2016) e268-9.

[8] Z. Bo, Y. Tian, Z. J. Han, S. Wu, S. Zhang, J. Yan, K. Cen, K. Ostrikov, Tuneable fluidics within graphene nanogaps for water purification and energy storage, Nanoscale Horiz. 2 (2017) 89-98.

[9] S. Zhu, Y. Song, J. Wang, H. Wan, Y. Zhang, Y. Ning, B. Yang, Photoluminescence mechanism in graphene quantum dots: Quantum confinement effect and surface/edge state, Nano Today 13 (2017) $10-14$.

[10] D. Benetti, K. T. Dembele, J. Benavides, H. Zhao, S. Cloutier, I. Concina, A. Vomiero and F. Rosei, Functionalized multi-wall carbon nanotubes/ $/ \mathrm{TiO}_{2}$ composites as efficient photoanodes for dye sensitized solar cells, J. Mater. Chem. C 4 (2016) 3555-3562.

[11] A. F. Zedan, S. Moussa, J. Terner, G. Atkinson, M. S. El-Shall, Ultrasmall gold nanoparticles anchored to graphene and enhanced photothermal effects by Laser irradiation of gold nanostructures in graphene oxide solutions, ACS Nano 7 (2013) 627-636.

[12] N. T. Lan, D. T. Chi, N. X. Dinh, N. D. Hung, H. Lan, P. A. Tuan, L. H. Thang, N. N. Trung, N. Q. Hoa, T. Q. Huy, N. V. Quy, T.-T. Duong, V. N. Phan, A.-T. Le, Photochemical decoration of silver nanoparticles on graphene oxide nanosheets and their optical characterization, J. Alloys Compd.615 (2014) 843-848.

[13] T.-H. Kim, K.-B. Lee, J.-W. Choi, 3D graphene oxide-encapsulated gold nanoparticles to detect neural stem cell differentiation, Biomaterials 34 (2013) 8660-8670.

[14] M. Hamzah, M. Khenfouch, V. V. Srinivasu, The quenching of silver nanoparticles photoluminescence by graphene oxide: spectroscopic and morphological investigations, J. Mater. 
Sci.: Mater. Electron. 28 (2017) 1804-1811.

[15] B. Park, S. J. Kim, J. S. Sohn, M. S. Nam, S. Kang, S. C. Jun, Surface plasmon enhancement of photoluminescence in photo-chemically synthesized graphene quantum dot and Au nanosphere, Nano Res. 9 (2016) 1866-1875.

[16] X. Zheng, W. Chen, G. Wang, Y. Yu, S. Qin, J. Fang, F. Wang, X.-A. Zhang, The Raman redshift of graphene impacted by gold nanoparticles, AIP Adv. 5 (2015) 057133-7.

[17] Y. Wang, Z. Ni, H. Hu, Y. Hao, C. P. Wong, T. Yu, J. T. L. Thong, Z. X. Shen, Gold on graphene as a substrate for surface enhanced Raman scattering study, Appl. Phys. Lett. 97 (2010) 163111-3.

[18] X. Li, W. Cai, J. An, S. Kim, J. Nah, D. Yang, R. Piner, A. Velamakanni, I. Jung, E. Tutuc, S. K. Banerjee, L. Colombo, R. S. Ruoff, Large-area synthesis of high-quality and uniform graphene films on copper foils, Science 324 (2009) 1312-1314.

[19] B. B. Wang, K. Zheng, Q. J. Cheng, L. Wang, M. P. Zheng, K. Ostrikov, Formation and electron field emission of graphene films grown by hot filament chemical vapor deposition, Mater. Chem. Phys.144 (2104) 66-74.

[20] A. E. Rider, I. Levchenko, K. Ostrikov, Surface fluxes of Si and C adatoms at initial growth stages of SiC quantum dots, J. App. Phys. 101 (2007) 044306-7.

[21] B. Lu, T. Iimori, K. Sakamoto, K. Nakatsuji, F. Rosei, F. Komori, Fullerene on nitrogen-adsorbed $\mathrm{Cu}(001)$ nanopatterned surfaces: from preferential nucleation to layer-by-layer growth, J. Phys. Chem. C 112 (2008) 10187-10192.

[22] Z. Bo, S. Mao, Z. J. Han, K. Cen, J. Chen, K. Ostrikov, Emerging energy and environmental applications of vertically-oriented graphenes, Chem. Soc. Rev.44 (2015) 2108-2121.

[23] K. Yu, P. Wang, G. Lu, K.-H. Chen, Z. Bo, J. Chen, Patterning vertically oriented graphene sheets for nanodevice applications, J. Phys. Chem. Lett. 2 (2011) 537-542.

[24] I. Levchenko, M. Keidar, S. Xu, H. Kersten and K. Ostrikov, Low-temperature plasmas in carbon nanostructure synthesis, J. Vac. Sci. Technol. B 31 (2013) 050801-16.

[25] B. B. Wang, K. Ostrikov, Z. L. Tsakadze, S. Xu, Analysis of photoluminescence background of Raman spectra of carbon nanotips grown by plasma-enhanced chemical vapor deposition, J. Appl. Phys. 106 (2009) 013315-7.

[26] B. Wang, K. Zheng, Q. Cheng, L. Wang, . Chen, G. Dong, Catalyst-free growth mechanism and structure of graphene-like nanosheets formed by hot-filament CVD, Chem. Vap. Deposition 20 (2014) 345-351.

[27] D. H. Seo, S. Kumar, K. Ostrikov, Control of morphology and electrical properties of self-organized graphenes in a plasma, Carbon 49 (2011) 4331-4339.

[28] I. Childres, L. A. Jauregui, Y. P. Chen, Raman spectra and electron-phonon coupling in disordered graphene with gate-tunable doping, J. Appl. Phys.116 (2014) 233101-7. 
[29] L. M. Malard, M. A. Pimenta, G. Dresselhaus, M. S. Dresselhaus, Raman spectroscopy in grapheme, Phys. Reports 473 (2005) 51-87.

[30] Z. Zafar, Z. H. Ni, X. Wu, Z. X. Shi, H. Y. Nan, J. Bai, L. T. Sun, Evolution of Raman spectra in nitrogen doped grapheme, Carbon 61 (2013) 57-62.

[31] T. van der Laan, S. Kumar, K. Ostrikov, Water-mediated and instantaneous transfer of graphene grown at $220{ }^{\circ} \mathrm{C}$ enabled by a plasma, Nanoscale 7 (2015) 20564-20570.

[32] C. Casiraghi, S. Pisana, K. S. Novoselov, A. K. Geim, A. C. Ferrari, Raman fingerprint of charged impurities in graphene, Appl. Phys. Lett. 91 (2007) 233108-3.

[33] C. D. Wagner, W. M. Riggs, L. E. Davis, J. F. Moulder, G. E. Muilenberg, Handbook of X-ray Photoelectron Spectroscopy, Perkin-Elmer Corp., Physical Electronics Division, USA, 1979.

[34] B. B. Wang, R. W. Shao, X. L. Qu, I. Levchenko, M. Keidar, X. X. Zhong, K. Zheng, S. Xu, K. Ostrikov, Pillar BNCO nanoflake/nanorod hybrid networks synthesized by plasma-enhanced hot filament CVD: Structure and photoluminescence, Carbon 118 (2017) 615-624.

[35] I. Levchenko, O. Baranov, Simulation of island behavior in discontinuous film growth, Vacuum 72 (2003) 205-210.

[36] S. C. Lee, H. J. Seo, G. Y. Han, Hydrogen production by catalytic decomposition of methane over carbon black catalyst at high temperatures, Korean J. Chem. Eng. 30 (2013) 1716-1721.

[37] D. Takagi, Y. Kobayashi, H. Hibino, S. Suzuki, Y. Homma, Mechanism of gold-catalyzed carbon material growth, Nano Lett. 8 (2008) 832-835.

[38] H. Okamoto, T. B. Massalski, The Au-C (goldecarbon) system, Bulletin of Alloy Phase Diagram, 5 (1984) 378-379.

[39] B. B. Wang, K. Ostrikov, T. van der Laan, K. Zheng, J. J. Wang, Y. P. Yan, X. J. Quan, Carbon nanorods and graphene-like nanosheets by hot filament CVD: growth mechanisms and electron field emission, J. Mater. Chem. C 1 (2013) 7703-7708.

[40] K. Ostrikov, H. Mehdipour, Rapid, simultaneous activation of thin nanowire growth in low-temperature, low-pressure chemically active plasmas, J. Mater. Chem. 21 (2011) 8183-8191.

[41] I. Levchenko, K. Ostrikov, A. B. Murphy, Plasma-deposited Ge nanoisland films on Si: is StranskiKrastanow fragmentation unavoidable, J. Phys. D: Appl. Phys. 41 (2008) 092001-5.

[42] K. Zheng, C. Wang, Y.-Q. Cheng, Y. Yue, X. Han, Z. Zhang, Z. Shan, S. X. Mao, M. Ye, Y. Yin, E. Ma, Electron-beam-assisted superplastic shaping of nanoscale amorphous silica, Nat. Commun. 1 (2010) 24-8.

[43] L. Wang, J. Teng, P. Liu, A. Hirata, E. Ma, Z. Zhang, M. Chen, X. Han, Grain rotation mediated by grain boundary dislocations in nanocrystalline platinum, Nat. Commun. 5 (2014) 4402-7.

[44] P. Liu, S. C. Mao, L. H. Wang, X. D. Han, Z. Zhang, Direct dynamic atomic mechanisms of 
strain-induced grain rotation in nanocrystalline, textured, columnar-structured thin gold films, Scripta Mater. 64 (2011) 343-346.

[45] A. Malesevic, R. Vitchev, K. Schouteden, A. Volodin, L. Zhang, G. V. Tendeloo, A. Vanhulsel, 1 C. V. Haesendonck, Synthesis of few-layer graphene via microwave plasma-enhanced chemical vapour deposition, Nanotechnol. 19 (2008) 305604-6.

[46] G. T. Boyd, Z. H. Yu, Y. R. Shen, Photoinduced luminescence from the noble metals and its enhancement on roughened surfaces, Phys. Rew. B 12 (1986) 7923-7936.

[47] M. R. Beversluis, A. Bouhelier, L. Novotny, Continuum generation from single gold nanostructures through near-field mediated intraband transitions, Phys. Rev. B 68 (2003) 115433-10.

[48] F. Bonaccorso, Z. Sun, T. Hasan and A. C. Ferrari, Graphene photonics and optoelectronics, Nat. Photonics 4 (2010) 611-622.

[49] T. Gokus, R. R. Nair, A. Bonetti, M. Böhmler, A. Lombardo, K. S. Novoselov, A. K. Geim, A. C. Ferrari, A. Hartschuh, Making graphene luminescent by oxygen plasma treatment, ACS Nano 3 (2009) 3963-3968.

[50] G. Eda, Y.-Y. Lin, C. Mattevi, H. Yamaguchi, H.-A. Chen, I-S. Chen, C.-W. Chen, M. Chhowalla, Blue photoluminescence from chemically derived graphene oxide, Adv. Mater. 22 (2010) 505-509.

[51] Y. Iwano, T. Kittaka, H. Tabuchi, M. Soukawa, S. Kunitsugu, K. Takarabe, K. Itoh, Study of amorphous carbon nitride films aiming at white light emitting devices, Jap. J. Appl. Phys. 47 (2008) $7842-7844$.

[52] E. Dulkeith, T. Niedereichholz, T. A. Klar, and J. Feldmann, G. von Plessen, D. I. Gittins, K. S. Mayya, F. Caruso, Plasmon emission in photoexcited gold nanoparticles, Phys. Rev. B 70 (2004) 205424-4.

[53] S. G. Zhang, L. Wen, J. L. Li, F. L. Gao, X. W. Zhang, L. H. Li, G. Q. Li, Plasmon-enhanced ultraviolet photoluminescence from highly ordered $\mathrm{ZnO}$ nanorods/graphene hybrid structure decorated with Au nanospheres, J. Phys. D: Appl. Phys., 47 (2014) 495103-6.

[54] A. Hoggard, L.-Y. Wang, L. Ma, Y. Fang, G. You, J. Olson, Z. Liu, W.-S. Chang, P. M. Ajayan, S. Link, Using the plasmon linewidth to calculate the time and efficiency of electron transfer between gold nanorods and graphene, ACS Nano 7 (2013) 11209-11217.

[55] T. Pons, I. L. Medintz, K. E. Sapsford, S. Higashiya, A. F. Grimes, D. S. English, H. Mattoussi, On the quenching of semiconductor quantum dot photoluminescence by proximal gold nanoparticles, Nano Lett. 7 (2007) 3157-3164.

[56] J. R. Lakowicz, Y. Shen, S. D'Auria, J. Malicka, J. Fang, Z. Gryczynski, I. Gryczynski, Radiative Decay Engineering, Anal. Biochem. 301 (2002) 261-277.

[57] L. H. Wang, C. Y. Song, L. Zhang, D. Zhu, Plasmon Nanomaterials and Their Applications in Biology and Medicine Science, Plasmon Nanomaterials and Their Applications in Biology and 
Medicine Science, Beijing: Science Press, 2017 (in Chinese).

[58] E. Perevedentseva, N. Melnik, C.-Y. Tsai, Y.-C. Lin, M. Kazaryan, C.-L. Cheng, Effect of surface adsorbed proteins on the photoluminescence of nanodiamond, J. Appl. Phys. 109 (2011) 034704-6.

[59] Y. A. Wang, J. J. Li, H. Chen, X. Peng, Stabilization of inorganic nanocrystals by organic dendrons, J. Am. Chem. Soc. 124 (2002) 2293-2298.

[60] S. Chaudhary, J. H. Kim, K. V. Singh, M. Ozkan, Fluorescence microscopy visualization of single-walled carbon nanotubes using semiconductor nanocrystals, Nano lett. 4 (2004) 2415-2419.

[61] J. Fang, I. Levchenko, T. van der Laan, S. Kumar, K. K. Ostrikov, Multipurpose nanoporous alumina-carbon nanowall bi-dimensional nano-hybrid platform via catalyzed and catalyst-free plasma CVD, Carbon 78 (2014) 627-632.

[62] S. Kumar, I. Levchenko, K. K. Ostrikov, J. A. McLaughlin, Plasma-enabled, catalyst-free growth of carbon nanotubes on mechanically-written Si features with arbitrary shape, Carbon 50 (2012) 325-329

[63] I. Levchenko, K. Bazaka, M. Keidar, S. Xu and J. Fang, Hierarchical multi-component inorganic metamaterials: intrinsically driven self-assembly at nanoscale, Adv. Mater. 2017 (in press, DOI: 10.1002/adma.201702226)

[64] K. Ostrikov, I. B. Denysenko, S. V. Vladimirov, S. Xu, H. Sugai, M. Y. Yu, Low-pressure diffusion equilibrium of electronegative complex plasmas, Phys. Rev. E 67 (2003), 056408. 